\title{
Structural shape parametric optimization for an internal structural-acoustic problem
}

\author{
Christian Soize $^{\text {a, } *}$, Jean-Christophe Michelucci ${ }^{\text {a }}$ \\ a ONERA, Structural Dynamics and Coupled Systems Department, BP 72, 92322 Châtillon cedex, France
}

Received 11 May 1999; revised 28 February 2000; accepted 9 March 2000

\begin{abstract}
A structural shape optimization problem, with respect to the structural aspect ratio is developed in the context of an axisymmetric structural-acoustic system, consisting of an elastic dome coupled with an internal acoustic cavity, is analyzed in the low- and medium-frequency ranges. The dome is a thin shell considered as a three-dimensional continuum with a dissipative constitutive equation. The internal $\mathrm{f}$ uid is a dissipative acoustic $\mathrm{fl}$ id. The dome is submitted to an external wall pressure fi ld modeled by a stochastic fi ld. The cost function is related to the pressure fi ld over an internal axisymmetric observation surface inside the acoustic cavity. We are interested in minimizing the internal noise over the observation surface with respect to the structural aspect ratio def ning the geometric shape of the dome. This paper develops an analysis of the structural-acoustic shape optimization problem to determine wheter or not there exist values of the dome aspect ratio for which the internal noise is a minimum. The frequency response functions of the structuralacoustic system are calculated to construct the cost function. In this context, the Fourier series expansions of the structural displacement $\mathrm{f}$ eld and the internal $\mathrm{f}$ uid velocity potential are carried out with respect to the polar angle variable. For each $\mathrm{f}$ xed circumferential wave number, a reduced matrix model is constructed using the structural modes of the structure in vacuo and the acoustic modes of the internal acoustic cavity with rigid wall. The structural modes and the acoustic modes are computed by the f nite element method. The optimization parameter is the aspect ratio of the structure. The analysis presented shows that the structural shape optimization problem of the dome with respect to its aspect ratio parameter has a clear solution which minimizes internal noise in the low- and medium-frequency ranges.
\end{abstract}

shape optimization / structural acoustics / internal noise / acoustics / dynamics / vibration 


\section{Introduction}

This paper deals with a structural shape parametric optimization in the structural-acoustic area. The structure is an elastic dome constituted by an axisymmetric thin shell structure considered as a three-dimensional continuum with a dissipative constitutive equation. The shape of the dome is define by its aspect ratio which is a scalar parameter. We are interested in the optimization problem with respect to this scalar parameter and not in the general shape optimization [5]. The dome is coupled with an axisymmetric internal dissipative acoustic fl id. The dome is excited by an external random wall pressure f eld which is stationary in time, such as a wall pressure induced by a turbulent boundary layer due to an external $\mathrm{fl} \mathrm{w}$. The objective of this paper is only devoted to analyzing the inf uence of the dome curvature on the coupling mechanism between the structure and the internal acoustic cavity (see below). Consequently, the coupling effects of the structure with the unbounded external acoustic fuid is neglected in order to simplify the parametric analysis. It should be noted that the effects of the external $f$ uid on the structure have two effects $[7,12]$. The $\mathrm{f}$ rst one is an additional damping for the structure, induced by the acoustic radiation at inf nity in the external $\mathrm{f}$ uid. This additional damping, which depends on the frequency is smaller than the structural damping and, consequently, does not modify the coupling mechanism between the structure and the internal acoustic cavity. The second effect, which is induced by the external acoustic $\mathrm{f}$ uid, is an added mass for the structure. This added mass, which depends on the frequency, generally produces a decreasing of the eigenfrequencies of the structure in a vacuum when the external acoustic $\mathrm{f}$ uid is a liquid. This shift effect is significa $t$ for the frst structural eigenmodes in the LF range. When the modal density of the structure is high enough [10,17], which is the case for the superior part of the LF range and for the MF range of the structure under consideration, the modal density of the structure is not signif cantly modif ed, and def nitely not sufficie tly modifie to distort the performed analysis of the coupling mechanism between the structure and the internal acoustic cavity. The observation made on this axisymmetric structural-acoustic system is the internal pressure f eld over an internal axisymmetric observation surface inside the acoustic cavity. We are interested in minimizing the internal noise over the observation surface with respect to the geometric shape of the dome defi ed by its aspect ratio and consequently, the optimization parameter is the aspect ratio of the dome (axisymmetric structure).

The fundamental mechanism induced by the curvature of the dome on the coupling between the structure and the internal acoustic cavity, is the following. The structural membrane waves and the structural fexural waves in the dome are coupled by the curvature of the dome. Structural $\mathrm{f}$ exural waves excited by the external wall pressure are converted into structural membrane waves. These structural membrane waves induce a piston movement of the dome nose. This type of structural displacement is associated with a variation in volume of the internal acoustic cavity which induces a high level of internal noise (presently, the observation surface inside the acoustic cavity is not located in the near fiel of the wall but is located in the far fiel ). This paper develops an analysis of the structural-acoustic shape optimization problem to determine whether or not there exist values of the dome aspect ratio for which the internal noise is a minimum (without, however, developing an automatic optimization algorithm).

Because we are interested in the low- and mediumfrequency ranges and taking into account the fact that the dome geometry is not a 'simple shape geometry', the above parametric optimization problem is diff cult to solve by analytical methods $[3,7,9,13]$. This is why the numerical approach proposed in reference [12] is used to solve this internal structural-acoustic optimization problem. According to this reference, in the general case, the modal approach, which is perfectly adapted to the low-frequency range, cannot be extended to the mediumfrequency range [12]. However, because the structuralacoustic system considered is axisymmetric, a Fourier series expansion of the response can be made and therefore, the modal approach can still be used for the mediumfrequency range which is considered in the application presented in this paper. It should be noted that this method could not be used for a general three-dimensional structural-acoustic system. In addition, in reference [12], the formulation proposed for internal structural-acoustic systems in the low-frequency range differs from the formulation adapted to the medium-frequency range. Since we are not interested in calculation of the structuralacoustic modes and since we wish to use a single formulation for the optimization problem related to the low- and medium-frequency ranges, we chose to use the medium- 
frequency model presented in [12]. A reduced matrix model for each fi ed circumferential wave number is constructed by a Ritz-Galerkin projection based on the use of the structural modes of the structure in vacuo and the acoustic modes of the internal acoustic cavity with rigid wall. The structural modes and the acoustic modes are computed by the f nite element method.

Section 2 is devoted to (1) the geometric description of the three-dimensional axisymmetric structural-acoustic system, (2) its mechanical modeling and the associated three-dimensional boundary value problem, and (3) the Fourier series expansion and the two-dimensional boundary value problems associated with each fi ed circumferential wave number. In section 3, we give the variational formulation of the two-dimensional boundary value problem associated with a fxed circumferential wave number. Section 4 is devoted to the construction of the reduced matrix model using the Ritz-Galerkin method. In section 5, we defin a normalized power spectral density function of the observation, which is directly used to construct the cost function of the optimization problem. In section 6, we defi e the optimization problem and we present the method for constructing its solution. Finally, in section 7 we describe a complete numerical application.

\section{Three-dimensional axisymmetric structural-acoustic system boundary value problem}

\subsection{Structural-acoustic system geometry}

The geometry of the structural-acoustic system is defi ed in figu e 1. The internal acoustic fl id occupies a bounded domain $D_{F}$ of three-dimensional physical space $\mathbb{R}^{3}$ with boundary $\Sigma_{F}=\Sigma^{-} \cup \Sigma_{F}^{-} \cup \Sigma_{F}^{0}$ in which $\Sigma_{F}^{-}$and $\Sigma_{F}^{0}$ are rigid walls. The structure occupies a bounded domain $D_{S}$ of $\mathbb{R}^{3}$ with boundary $\Sigma_{S}=\Sigma^{-} \cup$ $\Sigma^{+} \cup \Sigma_{S}^{0}$ in which $\Sigma_{S}^{0}$ is a rigid wall whereas boundary $\Sigma^{-}$is the coupling interface between the elastic structure and the internal acoustic f uid. The unit normal to $\Sigma_{S}$ external to $D_{S}$ and the unit normal to $\Sigma_{F}$ external to $D_{F}$ are denoted as $\mathbf{n}$ and $\mathbf{n}^{\prime}$ respectively. Therefore, $\mathbf{n}=-\mathbf{n}^{\prime}$ on $\Sigma^{-}$. Space $\mathbb{R}^{3}$ is referenced to a cartesian reference system $\left(0, x_{1}, x_{2}, x_{3}\right)$ also written $(0, x, y, z)$. The generic point of $\mathbb{R}^{3}$ is denoted as $\mathbf{x}=\left(x_{1}, x_{2}, x_{3}\right)$. We introduce the cylindrical coordinates $(\theta, r, z) \in\left[0,2 \pi\left[\times \mathbb{R}^{+} \times \mathbb{R}\right.\right.$ such that $x_{1}=-r \sin \theta, x_{2}=r \cos \theta$ et $x_{3}=z$. The associated local cylindrical orthonormal basis is $\left(\mathbf{e}_{\theta}, \mathbf{e}_{r}, \mathbf{e}_{z}\right)$ as shown in figu e 2 . The coupled system is axisymmetric around axis $(0 z)$ as is the internal observation surface $\Sigma$. The generating plane is defi ed by $\{\mathbf{x} \mid x=0, y>0\}$. Parts $\Sigma^{+}$and $\Sigma^{-}$are denoted as $\Sigma^{i}$ for $i$ in $\{+,-\}$. The generatrix of $\Sigma^{i}$ is denoted as $\Gamma^{i}$ and is define by the function $z \mapsto R^{i}(z)$. The generatrix $\Gamma$ of internal observation surface $\Sigma$ is def ned by the function $z \mapsto R(z)$.

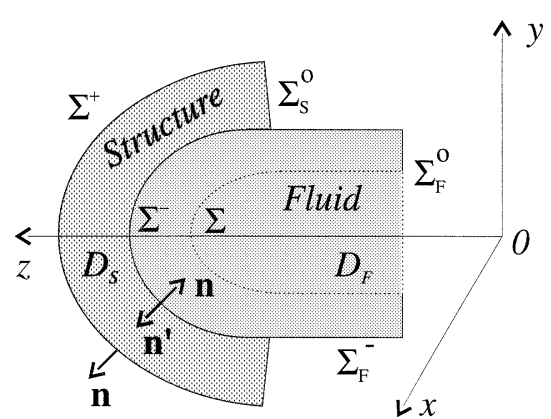

Figure 1. Geometry of the structural-acoustic system.

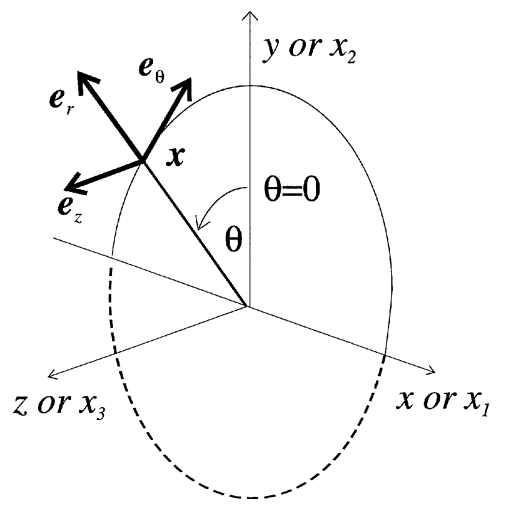

Figure 2. Associated local cylindrical orthonormal basis.

The generating planes of the structure and the internal acoustic flui are denoted as $P_{S}$ and $P_{F}$ respectively. Boundaries $\Sigma_{F}$ and $\Sigma_{S}^{o}$ are generated by curves $\Gamma_{F}$ and $\Gamma_{S}^{0}$ respectively. Let $s^{i}$ be the curvilinear abscissa of generatrix $\Gamma^{i}$ pointing positively in the direction of increasing $z$. We introduce the curvilinear measure $d s^{i}$ related to $\Gamma^{i}$ and measures $d \Gamma^{i}$ and $d \Sigma^{i}$ such that

$$
\begin{aligned}
d s^{i}(z) & =\left(1+\left(d R^{i}(z) / d z\right)^{2}\right)^{1 / 2} d z, \\
d \Gamma^{i} & =R^{i}(z) d s^{i}(z), \quad d \Sigma^{i}=R^{i}(z) d \theta d s^{i}(z) .
\end{aligned}
$$

For all $i$ in $\{+,-\}$ and all $\mathbf{x}$ in $\Sigma^{i}$, we defin a local physical reference system $\left(\mathbf{b}_{1}^{i}=\mathbf{e}_{\left.\theta\right|_{\Sigma^{i}}}, \mathbf{b}_{2}^{i}=\mathbf{n}^{i}, \mathbf{b}_{3}^{i}=\right.$ $\mathbf{e}_{\left.\theta\right|_{\Sigma^{i}}} \wedge \mathbf{n}^{i}$ ) attached to point $\mathbf{x}$ where $\mathbf{n}^{+}=\mathbf{n}$ on $\Sigma^{+}$ and $\mathbf{n}^{-}=-\mathbf{n}$ on $\Sigma^{-}$. The orthogonal $3 \times 3$ matrix transforming the local physical reference system into the local cylindrical reference system depends only on $z$ and is written as

$$
\begin{aligned}
{\left[\Theta^{i}(z)\right] } & =\left[\begin{array}{ccc}
1 & 0 & 0 \\
0 & \alpha^{i}(z) & \beta^{i}(z) \\
0 & -\beta^{i}(z) & \alpha^{i}(z)
\end{array}\right], \\
\alpha^{i}(z) & =\left(1+\left(d R^{i}(z) / d z\right)^{2}\right)^{-1 / 2}, \\
\beta^{i}(z) & =\alpha^{i}(z) \frac{d R^{i}(z)}{d z} .
\end{aligned}
$$




\subsection{Modeling and boundary value problem of the three-dimensional axisymmetric structural-acoustic system}

We consider linear vibrations of the structural-acoustic system around a static equilibrium position without prestresses and taken as reference conf guration. The formulation is written in the frequency domain $\omega$ (angular frequency in $\mathrm{rad} / \mathrm{s}$ ) for which the Fourier transform convention used is such that, if $t \mapsto f(t)$ is a function from $\mathbb{R}$ into $\mathbb{C}$, then its Fourier transform is written as $f(\omega)=\int_{\mathbb{R}} \mathrm{e}^{-i \omega t} f(t) d t$.

\subsubsection{External excitation}

The external mechanical excitation applied to the structure is the vector f eld $\mathbf{x} \mapsto \mathbf{f}(\mathbf{x}, \omega)=-p^{+}(\mathbf{x}, \omega) \mathbf{n}(\mathbf{x})$ def ned on $\Sigma^{+}$with values in $\mathbb{C}^{3}$.

\subsubsection{Internal acoustic flud}

We assume that there is no acoustic source inside acoustic fuid domain $D_{F}$. The internal $\mathrm{fl}$ id is a dissipative acoustic fl id. Let $\rho_{0}$ be the mass density and $c_{0}$ the speed of sound in the equilibrium state. The pressure fiel $p(\mathbf{x}, \omega)$ and the velocity potential $\psi(\mathbf{x}, \omega)$ are such that $[11,12]$

$$
\begin{aligned}
& p(\mathbf{x}, \omega)=-i \omega \rho_{0} \psi(\mathbf{x}, \omega)-\kappa \pi_{2}(\mathbf{u}), \\
& \kappa=\frac{\rho_{0} c_{0}^{2}}{\left|D_{F}\right|}, \quad \pi_{2}(\mathbf{u})=\int_{\Sigma^{-}}\left\langle\mathbf{u}(\mathbf{x}, \omega), \mathbf{n}^{\prime}(\mathbf{x})\right\rangle d \Sigma^{-},
\end{aligned}
$$

in which $i$ denotes the pure imaginary complex number, $\langle\mathbf{u}, \mathbf{v}\rangle=u_{1} v_{1}+u_{2} v_{2}+u_{3} v_{3},\left|D_{F}\right|=\int_{D_{F}} d \mathbf{x}$ is the volume of the internal acoustic cavity and where $\psi$ must satisfy the following constraint equation $\int_{D_{F}} \psi(\mathbf{x}, \omega) d \mathbf{x}=$ 0 . It should be noted that in this model, $\psi$ is not exactly a velocity potential because the velocity vector is written as $\mathbf{v}(\mathbf{x}, \omega)=(1+i \omega \tau) \nabla \psi(\mathbf{x}, \omega)$. The three-dimensional equations for the internal acoustic fl id are written as in [12]

$$
\begin{aligned}
& -\omega^{2} \frac{\rho_{0}}{c_{0}^{2}} \psi-i \omega \lambda_{0} \rho_{0} \nabla^{2} \psi-\rho_{0} \nabla^{2} \psi \\
& =-\frac{i \omega \kappa}{c_{0}^{2}} \pi_{2}(\mathbf{u}) \quad \text { in } D_{F}, \\
& \rho_{0}\left(1+i \omega \lambda_{0}\right) \frac{\partial \psi}{\partial \mathbf{n}}=i \omega \rho_{0}\langle\mathbf{u}, \mathbf{n}\rangle \text { on } \Sigma^{-}, \\
& \frac{\partial \psi}{\partial \mathbf{n}}=0 \quad \text { on } \Sigma_{F}^{o} \cup \Sigma_{F}^{-}, \\
& \int_{D_{F}} \psi d \mathbf{x}=0,
\end{aligned}
$$

in which $\lambda_{0}$ is a damping coeff cient which may depend on $\omega$. Constraint equation (10) shows that $\psi$ cannot be a constant $f$ eld.

\subsubsection{Structure}

The structure is an axisymmetric three-dimensional solid continuum with a linear viscoelastic constitutive equation without memory. Its mass density and its displacement $f$ eld with values in $\mathbb{C}^{3}$ are denoted as $\rho_{S}(\mathbf{x})>$ 0 and $\mathbf{u}(\mathbf{x}, \omega)$ respectively. The constitutive equation is written as in [12], $\sigma_{j k}(\mathbf{x}, \omega)=\sigma_{j k}^{e}(\mathbf{x}, \omega)+i \omega \sigma_{j k}^{d}(\mathbf{x}, \omega)$ in which $\sigma_{j k}$ is the stress tensor, $\sigma_{j k}^{e}(\mathbf{x}, \omega)=a_{j k h \ell}(\mathbf{x}) \times$ $\varepsilon_{h \ell}(\mathbf{u})$ is the elastic part of the stress tensor, $\sigma_{j k}^{d}(\mathbf{x}, \omega)=$ $b_{j k h \ell}(\mathbf{x}) \varepsilon_{h l}(\mathbf{u})$ is the damping part of the stress tensor and $\varepsilon_{j k}=\frac{1}{2}\left(\partial_{k} u_{j}+\partial_{j} u_{k}\right)$ is the linearized strain tensor in which $\partial_{k}$ denotes the partial derivative with respect to $x_{k}$. Elastic coefficie ts $a_{j k h \ell}(\mathbf{x})$ and damping coefficient $b_{j k h \ell}(\mathbf{x})$ are real and are assumed to be independent of $\omega$ (linear viscoelasticity without memory) in the context of the present shape optimization problem in order to simplify the formulation. These coeff cients depend on $\mathbf{x}$ and satisfy the usual symmetry and positivity properties. It is assumed that no external body force f eld is applied to the structure. In the cartesian reference system and for $j$ and $k$ in $\{1,2,3\}$, the elastodynamic equation is written as

$$
\begin{aligned}
& -\omega^{2} \rho_{S} u_{j}-\partial_{k} \sigma_{j k}(\mathbf{u})=0 \quad \text { in } D_{S}, \\
& u_{j}=0 \quad \text { on } \Sigma_{S}^{0}, \\
& \sigma_{j k}(\mathbf{u}) n_{k}=-p_{\Sigma^{-}} n_{k} \quad \text { on } \Sigma^{-}, \\
& \sigma_{j k}(\mathbf{u}) n_{k}=f_{j} \text { on } \Sigma^{+} .
\end{aligned}
$$

\subsection{Fourier series expansion and two-dimensional boundary value problem associated with each fi ed circumferential wave number}

Since the three-dimensional boundary value problem defi ed in section 2.2 is axisymmetric, a Fourier series expansion of the solution can be made with respect to polar angle $\theta$. This yields a sequence of two-dimensional problems indexed by the circumferential wave number denoted as $n$. For all $n$ and $n^{\prime}$ in $\mathbb{N}$, the orthogonality properties are written as

$$
\begin{aligned}
& \int_{0}^{2 \pi} \sin n \theta \cos n^{\prime} \theta d \theta=0, \\
& \int_{0}^{2 \pi} \sin n \theta \sin n^{\prime} \theta d \theta=\delta_{n n^{\prime}}\left(1-\delta_{0 n}\right) \pi, \\
& \int_{0}^{2 \pi} \cos n \theta \cos n^{\prime} \theta d \theta=\delta_{n n^{\prime}}\left(1+\delta_{0 n}\right) \pi,
\end{aligned}
$$

in which $\delta_{n n^{\prime}}=0$ if $n \neq n^{\prime}$ and $\delta_{n n^{\prime}}=1$ if $n=n^{\prime}$. 


\subsubsection{Structural displacement feld}

Since the coupled system is axisymmetric, for any fixe $r, z$ and $\omega$, function $\theta \mapsto \mathbf{u}(\theta, r, z, \omega)$ is periodic with period $2 \pi$ and has the following Fourier series expansion

$$
\begin{aligned}
u_{\theta}(\mathbf{x}, \omega)= & \sum_{n=0}^{+\infty}\left(U_{\theta}^{(n, s)}(r, z, \omega) \sin n \theta\right. \\
& \left.+U_{\theta}^{(n, a s)}(r, z, \omega) \cos n \theta\right), \\
u_{r}(\mathbf{x}, \omega)= & \sum_{n=0}^{+\infty}\left(U_{r}^{(n, s)}(r, z, \omega) \cos n \theta\right. \\
& \left.-U_{r}^{(n, a s)}(r, z, \omega) \sin n \theta\right), \\
& +\infty \\
u_{z}(\mathbf{x}, \omega)= & \sum_{n=0}^{+(n, s)}(r, z, \omega) \cos n \theta \\
& \left.-U_{z}^{(n, a s)}(r, z, \omega) \sin n \theta\right),
\end{aligned}
$$

in which $u_{\theta}, u_{r}$ and $u_{z}$ are the components of feld $\mathbf{u}$ in the cylindrical basis and where for $j$ in $\{\theta, r, z\}, n$ in $\mathbb{N}$ and $I$ in $\{s, a s\}, U_{j}^{(n, I)}(r, z, \omega)$ is the complex-valued fiel defi ed on $P_{S} \times \mathbb{R}$. Functions $U_{\theta}^{(0, s)}, U_{r}^{(0, a s)}$ and $U_{z}^{(0, a s)}$ are equal to zero. Introducing the vector feld $\mathbf{U}^{(n, I)}=\left(U_{\theta}^{(n, I)}, U_{r}^{(n, I)}, U_{z}^{(n, I)}\right)$, equations (18)-(20) can be rewritten as

$$
\mathbf{u}(\mathbf{x}, \omega)=\sum_{n=0}^{+\infty} \sum_{I \in\{s, a s\}}\left[F^{(n, I)}(\theta)\right] \mathbf{U}^{(n, I)}(r, z, \omega),
$$

in which, for all $\theta$ in $[0,2 \pi]$, matrices $\left[F^{(n, s)}(\theta)\right]$ and $\left[F^{(n, a s)}(\theta)\right]$ are def ned by equations (18)-(20). Since we have $\left[\Theta^{i}(z)\right]^{T}\left[F^{(n, I)}(\theta)\right]\left[\Theta^{i}(z)\right]=\left[F^{(n, I)}(\theta)\right]$, for any f xed point on surface $\Sigma^{+}$or $\Sigma^{-}$, structural displacement fiel $\mathbf{u}$ is also given by equation (21) if it is expressed in the local physical reference system.

\subsubsection{Velocity potential of the internal acoustic flu $d$}

The Fourier series expansion of periodic function $\theta \mapsto$ $\psi(\theta, r, z, \omega)$ with period $2 \pi$ is written as

$$
\begin{aligned}
\psi(\theta, r, z, \omega)= & \sum_{n=0}^{+\infty}\left(\Psi^{(n, s)}(r, z, \omega) \cos n \theta\right. \\
& \left.-\Psi^{(n, a s)}(r, z, \omega) \sin n \theta\right),
\end{aligned}
$$

where for $n \in \mathbb{N}$ and $I \in\{\mathrm{s}$, as $\}$, the $\mathbb{C}$-valued twodimensional feld $(r, z) \mapsto \Psi^{(n, I)}(r, z, \omega)$ is def ned on $P_{F} \times \mathbb{R}$. Functions $\Psi^{(0, a s)}$ are equal to zero. Equation (22) can be rewritten as

$$
\psi(\mathbf{x}, \omega)=\sum_{n=0}^{+\infty} \sum_{I \in\{s, a s\}} F^{(n, I)}(\theta) \Psi^{(n, I)}(r, z, \omega),
$$

with $F^{(n, s)}(\theta)=\cos n \theta$ and $F^{(n, a s)}(\theta)=-\sin n \theta$. Let $\boldsymbol{\Psi}^{(n, s)}$ and $\boldsymbol{\Psi}^{(n, a s)}$ be the vector-valued f elds def ned by $\boldsymbol{\Psi}^{(n, s)}=\left(-\frac{n}{r} \Psi^{(n, s)}, \partial_{r} \Psi^{(n, s)}, \partial_{z} \Psi^{(n, s)}\right)$ and $\boldsymbol{\Psi}^{(n, a s)}=$ $\left(\frac{n}{r} \Psi^{(n, a s)},-\partial_{r} \Psi^{(n, a s)},-\partial_{z} \Psi^{(n, a s)}\right)$, depending on variables $(r, z, \omega)$. Substituting equations (21) and (23) into equation (5) and using equations (15)-(17) yields

$$
\begin{aligned}
p(\mathbf{x}, \omega)= & p^{(0)}(r, z, \omega)+\sum_{n=1}^{+\infty}\left(p^{(n, s)}(r, z, \omega) \cos n \theta\right. \\
& \left.-p^{(n, a s)}(r, z, \omega) \sin n \theta\right), \\
p^{(0)}(r, z, \omega)= & -i \omega \rho_{0} \Psi^{(0, s)}(r, z, \omega) \\
& -\kappa \pi_{2}\left(\mathbf{U}^{(0, s)}+\mathbf{U}^{(0, a s)}\right) \quad \text { if } n=0, \\
p^{(n, I)}(r, z, \omega)= & -i \omega \rho_{0} \Psi^{(n, I)}(r, z, \omega) \quad \text { if } n>0 .
\end{aligned}
$$

Substituting equations (21) and (23) into equations (7)(14) and using equations (15)-(17) yields a sequence of two-dimensional problems indexed by $n$ whose variational formulation is derived below.

\section{Variational formulation of the two-dimensional boundary value problem associated with a f xed circumferential wave number}

The admissible function space of displacement f eld $\mathbf{U}^{(n, I)}$ is the complex vector space $\mathbb{W}_{U}$ of 'suff ciently differentiable' function $\mathbf{U}$ def ned on $P_{S}$ with values in $\mathbb{C}^{3}$ such that $\mathbf{U}=0$ on $\Gamma_{S}^{0}$. The admissible function space of velocity potential $\Psi^{(n, I)}$ is the complex vector space $\mathbb{W} \Psi$ of 'suff ciently differentiable' function $\Psi$ def ned on $P_{F}$ with values in $\mathbb{C}$ such that $\partial \Psi / \partial \mathbf{n}=0$ on $\Gamma_{F}$ and $\int_{P_{F}} \Psi(r, z) r d r d z=0$. For a f xed circumferential wave number $n$ and $\omega$ fixe in $\mathbb{R}$, given $f^{(n, a)}$ and $f^{(n, a s)}$, the variational formulation of the two-dimensional problem indexed by $n$ consists in fnding $\mathbf{U}^{(n, I)}$ denoted as $\mathbf{U}$ in $\mathbb{W}_{U}$ and $\Psi^{(n, I)}$ denoted as $\Psi$ in $\mathbb{W}_{\Psi}$ such that, for all $\delta \mathbf{U}$ in $\mathbb{W}_{U}$ and for all $\delta \Psi$ in $\mathbb{W}_{\Psi}$, we have

$$
\begin{aligned}
& -\omega^{2} m_{S}^{(n)}(\mathbf{U}, \delta \mathbf{U})+i \omega c_{S}^{(n)}(\mathbf{U}, \delta \mathbf{U}) \\
& +i \omega a_{F}^{(n)}(\Psi, \delta \mathbf{U})+k_{S}^{(n)}(\mathbf{U}, \delta \mathbf{U}) \\
& +\kappa j^{(n)}(\mathbf{U}, \delta \mathbf{U})=f^{(n, I)}(\omega ; \delta \mathbf{U}), \\
& -\omega^{2} m_{F}^{(n)}(\Psi, \delta \Psi)+i \omega c_{F}^{(n)}(\omega ; \Psi, \delta \Psi) \\
& -i \omega a_{F}^{(n)}(\delta \Psi, \mathbf{U})+k_{F}^{(n)}(\Psi, \delta \Psi)=0,
\end{aligned}
$$

in which the mass, dissipation and stiffness structural bilinear forms are defi ed by

$$
\begin{aligned}
m_{S}^{(n)}(\mathbf{U}, \delta \mathbf{U})= & \left(1+\delta_{0 n}\right) \pi \\
& \times \int_{P_{S}} \rho_{S}\langle\mathbf{U}, \delta \mathbf{U}\rangle r d r d z,
\end{aligned}
$$




$$
\begin{aligned}
c_{S}^{(n)}(\mathbf{U}, \delta \mathbf{U})= & \left(1+\delta_{0 n}\right) \pi \int_{P_{S}} \sigma_{j k}^{(n, I), d}(\mathbf{U}) \\
& \times \varepsilon_{j k}^{(n, I)}(\delta \mathbf{U}) r d r d z, \\
k_{S}^{(n)}(\mathbf{U}, \delta \mathbf{U})= & \left(1+\delta_{0 n}\right) \pi \int_{D_{S}} \sigma_{j k}^{(n, I), e}(\mathbf{U}) \\
& \times \varepsilon_{j k}^{(n, I)}(\delta \mathbf{U}) r d r d z,
\end{aligned}
$$

and where $\varepsilon^{(n, I)}, \sigma^{(n, I), d}$ and $\sigma^{(n, I), e}$ denote the restriction of the strain tensor, the dissipative part and the elastic part of the stress tensor to $\mathbb{W}_{U}$. Concerning the internal acoustic fl id, we have

$$
\begin{aligned}
& m_{F}^{(n)}(\Psi, \delta \Psi)=\left(1+\delta_{0 n}\right) \pi \frac{\rho_{0}}{c_{0}^{2}} \int_{P_{F}} \Psi \delta \Psi r d r d z, \\
& c_{F}^{(n)}(\omega ; \Psi, \delta \Psi)=\lambda_{0}(\omega) k_{F}^{(n)}(\Psi, \delta \Psi), \\
& k_{F}^{(n)}(\Psi, \delta \Psi)=\left(1+\delta_{0 n}\right) \pi \rho_{0} \int_{P_{F}}\langle\Psi, \delta \Psi\rangle r d r d z,
\end{aligned}
$$

in which vectors $\boldsymbol{\Psi}$ and $\delta \boldsymbol{\Psi}$ are the vectors associated with $\Psi$ and $\delta \Psi$ as define section 2.3.2. The fluid structure coupling bilinear form $a_{F}^{(n)}$ and the bilinear form $j^{(n)}$ are define by

$$
\begin{aligned}
& a_{F}^{(n)}(\Psi, \delta \mathbf{U})=-\left(1+\delta_{0 n}\right) \pi \rho_{0} \int_{\Gamma^{-}} \Psi\langle\delta \mathbf{U}, \mathbf{n}\rangle d \Gamma^{-}, \\
& j^{(n)}(\mathbf{U}, \delta \mathbf{U})=\delta_{0 n} 4 \pi^{2} \pi_{2}^{(n)}(\mathbf{U}) \pi_{2}^{(n)}(\delta \mathbf{U}),
\end{aligned}
$$

with

$$
\pi_{2}^{(n)}(\mathbf{U})=-\int_{\Gamma^{-}}\langle\mathbf{U}, \mathbf{n}\rangle d \Gamma^{-},
$$

where $\langle\mathbf{U}, \mathbf{n}\rangle$ denotes the component of vector $\mathbf{U}$ along normal $\mathbf{n}$ on generatrix $\Gamma^{+}$. The linear form related to the excitation is defi ed by

$$
\begin{aligned}
f^{(n, I)}(\omega ; \delta \mathbf{U})= & -\int_{\Gamma^{+}} \int_{0}^{2 \pi} p^{+}(\theta, z, \omega) F^{(n, I)}(\theta) d \theta \\
& \times\langle\delta \mathbf{U}, \mathbf{n}\rangle(z) d \Gamma^{+}(z) .
\end{aligned}
$$

\section{Symmetric reduced matrix model}

As explained in section 1, for each fi ed circumferential wave number $n$, a symmetric reduced matrix model of equations (26) and (27) is constructed using the Ritz-Galerkin projection on a fi ite dimension subspace spanned by a set of structural modes of the structure in vacuo and a set of acoustic modes of the internal acoustic cavity with rigid wall. We then have a sequence of reduced matrix models indexed by $n$.

\subsection{Structural modes of the structure in vacuo}

For each fi ed $n$, the structural modes of the structure in vacuo are constructed by f nding the eigenvalues $\lambda=$ $\omega^{2}$ and the associated eigenfunctions $\mathbf{U} \in \mathbb{W}_{U}$ such that, for all $\delta \mathbf{U}$ in $\mathbb{W}_{U}$,

$$
k_{S}^{(n)}(\mathbf{U}, \delta \mathbf{U})=\lambda m_{S}^{(n)}(\mathbf{U}, \delta \mathbf{U})
$$

The spectrum of the eigenvalue problem def ned by equation (38) is the countable set $\lambda_{S, \alpha}^{(n)}=\left(\omega_{S, \alpha}^{(n)}\right)^{2}$ with $\alpha=$ $1,2, \ldots$ such that $0<\omega_{S, 1}^{(n)} \leqslant \omega_{S, 2}^{(n)} \leqslant \cdots$ and the associated real-valued eigenfunctions $\mathbf{U}_{\alpha}^{(n)}$ constitute a complete orthogonal set in $\mathbb{W}_{U}$. The normalization of the eigenfunctions are chosen such that $m_{S}^{(n)}\left(\mathbf{U}_{\alpha}^{(n)}, \mathbf{U}_{\alpha^{\prime}}^{(n)}\right)=$ $\mu_{S} \delta_{\alpha \alpha^{\prime}}$ in which $\mu_{S}=\int_{D_{S}} \rho_{S} d \mathbf{x}$ is the total structural mass. We keep only eigenvectors $\mathbf{U}_{\alpha}^{(n)}$ whose associated eigenfrequencies $\omega_{S, \alpha}^{(n)}$ lie in the frequency band of analysis denoted as $B_{0}$ and which are such that $\int_{\Gamma^{-}}\left\langle\mathbf{U}_{\alpha}^{(n)}, \mathbf{n}\right\rangle^{2} d \Gamma^{-} \neq 0$. Since there is no internal acoustic excitation but only an external structural excitation, it should be noted that if $\int_{\Gamma^{-}}\left\langle\mathbf{U}_{\alpha}^{(n)}, \mathbf{n}\right\rangle^{2} d \Gamma^{-}=0$, then eigenfunction $\mathbf{U}_{\alpha}^{(n)}$ has a contribution to the reponse of the structural displacement but no contribution to the pressure response of the internal acoustic cavity (we recall that the cost function of the optimization problem is formulated only in terms of the internal pressure feld). For each fi ed $n$, we denote the index set of such eigenfunctions as $\mathcal{J}_{S}^{(n)}=\left\{1, \ldots, N_{S}^{(n)}\right\}$. Consequently, vector fiel $\mathbf{U}$ as a solution of equations (26) and (27) is written as

$$
\mathbf{U}^{(n)}(r, z) \simeq \sum_{\alpha=1}^{N_{S}^{(n)}} X_{S, \alpha}^{(n)} \mathbf{U}_{\alpha}^{(n)}(r, z)
$$

\subsection{Acoustic modes of the internal acoustic cavity with rigid wall}

For each fi ed $n$, the acoustic modes of the internal acoustic cavity with rigid wall are constructed by f nding the eigenvalues $\lambda=\omega^{2}$ and the associated eigenfunctions $\Psi \in \mathbb{W}_{\Psi}$ such that, for all $\delta \Psi$ in $\mathbb{W}_{\Psi}$,

$$
k_{F}^{(n)}(\Psi, \delta \Psi)=\lambda m_{F}^{(n)}(\Psi, \delta \Psi) .
$$

The spectrum of the eigenvalue problem def ned by equation (40) is the countable set $\lambda_{F, \beta}^{(n)}=\left(\omega_{F, \beta}^{(n)}\right)^{2}$ with $\beta=$ $1,2, \ldots$, such that $0<\omega_{F, 1}^{(n)} \leqslant \omega_{F, 2}^{(n)} \leqslant \cdots$ and the associated real-valued eigenfunctions $\Psi_{\beta}^{(n)}$ constitute a complete orthogonal set in $\mathbb{W}_{\Psi}$. The normalization of the eigenfunctions is chosen such that $m_{F}^{(n)}\left(\Psi_{\beta}^{(n)}, \Psi_{\beta^{\prime}}^{(n)}\right)=$ 
$\left(\mu_{F} / c_{0}^{2}\right) \delta_{\beta \beta^{\prime}}$ in which $\mu_{F}=\int_{D_{F}} \rho_{0} d \mathbf{x}=\rho_{0}\left|D_{F}\right|$ is the total mass of the acoustic fl id. We keep only eigenvectors $\Psi_{\beta}^{(n)}$ whose associated eigenfrequencies $\omega_{F, \beta}^{(n)}$ lie in frequency band $B_{0}$ and such that there is an index $\alpha$ in $\mathcal{J}_{S}^{(n)}$ such that $a_{F}^{(n)}\left(\Psi_{\beta}^{(n)}, \mathbf{U}_{\alpha}^{(n)}\right) \neq 0$. If there is no such index $\alpha$, eigenfunction $\Psi_{\beta}^{(n)}$ has no contribution to the internal acoustic pressure (see section 4.1). For each fixe $n$, we denote the index set of such eigenfunctions as $\mathcal{J}_{F}^{(n)}=\left\{1, \ldots, N_{F}^{(n)}\right\}$. Consequently, f eld $\Psi$ of equations (26) and (27) is written as

$$
\Psi^{(n)}(r, z) \simeq \sum_{\beta=1}^{N_{F}^{(n)}} X_{F, \beta}^{(n)} \Psi_{\beta}^{(n)}(r, z) .
$$

\subsection{Reduced matrix model for each circumferential wave number}

For each fi ed $n$, the restriction of equations (26) and (27) to the subspaces of $\mathbb{W}_{U}$ and $\mathbb{W}_{\Psi}$ spanned by the fi ite families $\left\{\mathbf{U}_{1}^{(n)}, \ldots, \mathbf{U}_{N_{S}^{(n)}}^{(n)}\right\}$ and $\left\{\Psi_{1}^{(n)}, \ldots, \Psi_{N_{F}^{(n)}}^{(n)}\right\}$ respectively yields the reduced matrix model for circumferential wave number $n$ :

$$
\begin{aligned}
& \left(-\omega^{2}\left[\begin{array}{cc}
{\left[M_{S}^{(n)}\right]} & {\left[O_{S F}\right]} \\
{\left[O_{F S}\right]} & -\left[M_{F}^{(n)}\right]
\end{array}\right]\right. \\
& +i \omega\left[\begin{array}{cc}
{\left[C_{S}^{(n)}\right]} & {\left[A_{F}^{(n)}\right]} \\
{\left[A_{F}^{(n)}\right]^{T}} & -\left[C_{F}^{(n)}\right]
\end{array}\right] \\
& +\left[\begin{array}{ll}
{\left[K_{S}^{(n)}\right]} & {\left[O_{S F}\right]} \\
{\left[O_{F S}\right]} & -\left[K_{F}^{(n)}\right]
\end{array}\right] \\
& \left.+\kappa\left[\begin{array}{ll}
{\left[J^{(n)}\right]} & {\left[O_{S F}\right]} \\
{\left[O_{F S}\right]} & {\left[O_{F F}\right]}
\end{array}\right]\right)\left[\begin{array}{l}
\mathbf{X}_{S}^{(n, I)}(\omega) \\
\mathbf{X}_{F}^{(n, I)}(\omega)
\end{array}\right] \\
& =\left[\begin{array}{cc}
\mathbf{Y}_{S}^{(n, I)}(\omega) \\
\mathbf{0}
\end{array}\right],
\end{aligned}
$$

in which $\mathbf{X}_{S}^{(n, I)}=\left(X_{S, 1}^{(n)}, \ldots, X_{S, N_{S}^{(n)}}^{(n)}\right)$ and $\mathbf{X}_{F}^{(n, I)}=$ $\left(X_{F, 1}^{(n)}, \ldots, X_{F, N_{F}^{(n)}}^{(n)}\right)$ and with

$$
\begin{aligned}
{\left[M_{S}^{(n)}\right]_{\alpha \alpha^{\prime}} } & =m_{S}^{(n)}\left(\mathbf{U}_{\alpha}^{(n)}, \mathbf{U}_{\alpha^{\prime}}^{(n)}\right)=\mu_{S} \delta_{\alpha \alpha^{\prime}}, \\
{\left[C_{S}^{(n)}\right]_{\alpha \alpha^{\prime}} } & =2 \mu_{S} \xi_{S, \alpha}^{(n)} \omega_{S, \alpha}^{(n)} \delta_{\alpha \alpha^{\prime}}, \\
{\left[K_{S}^{(n)}\right]_{\alpha \alpha^{\prime}} } & =k_{S}^{(n)}\left(\mathbf{U}_{\alpha}^{(n)} \mathbf{U}_{\alpha^{\prime}}^{(n)}\right)=\mu_{S}\left(\omega_{S, \alpha}^{(n)}\right)^{2} \delta_{\alpha \alpha^{\prime}}, \\
{\left[M_{F}^{(n)}\right]_{\beta \beta^{\prime}} } & =m_{F}^{(n)}\left(\Psi_{\beta}^{(n)}, \Psi_{\beta^{\prime}}^{(n)}\right)=\left(\mu_{F} / c_{0}^{2}\right) \delta_{\beta \beta^{\prime}}, \\
{\left[C_{F}^{(n)}(\omega)\right]_{\beta \beta^{\prime}} } & =c_{F}^{(n)}\left(\Psi_{\beta}^{(n)}, \Psi_{\beta^{\prime}}^{(n)}\right) \\
& =2\left(\mu_{F} / c_{0}^{2}\right) \xi_{F, \beta}^{(n)} \omega_{F, \beta}^{(n)} \delta_{\beta \beta^{\prime}},
\end{aligned}
$$

$$
\begin{aligned}
{\left[K_{F}^{(n)}\right]_{\beta \beta^{\prime}} } & =k_{F}^{(n)}\left(\Psi_{\beta}^{(n)}, \Psi_{\beta^{\prime}}^{(n)}\right) \\
& =\left(\mu_{F} / c_{0}^{2}\right)\left(\omega_{F, \beta}^{(n)}\right)^{2} \delta_{\beta \beta^{\prime}}, \\
{\left[A_{F}^{(n)}\right]_{\alpha^{\prime} \beta} } & =a_{F}^{(n)}\left(\Psi_{\beta}^{(n)}, \mathbf{U}_{\alpha^{\prime}}^{(n)}\right), \\
{\left[J^{(n)}\right]_{\alpha \alpha^{\prime}} } & =j^{(n)}\left(\mathbf{U}_{\alpha}^{(n)}, \mathbf{U}_{\alpha^{\prime}}^{(n)}\right) .
\end{aligned}
$$

From equation (35), we deduce that $\left[J^{(n)}\right]=[0]$ for all $n \geqslant 1$. In the context of the present shape optimization problem and in order to simplify the formulation, we assume that the dissipation structural bilinear form is diagonalized by the eigenfunctions $\mathbf{U}_{\alpha}^{(n)}$. According to the eigenfunction properties introduced in sections 4.1 and 4.2, the coupling matrix is such that, for all $\mathbf{X}_{F} \in$ $\mathbb{C}^{N_{F}^{(n)}},\left[A_{F}^{(n)}\right] \mathbf{X}_{F}=0 \Rightarrow \mathbf{X}=0$. Finally, the generalized external structural forces $\mathbf{Y}_{S}^{(n, I)}=\left(Y_{S, 1}^{(n, I)}, \ldots, Y_{S, N_{S}^{(n)}}^{(n, I)}\right)$ are such that

$$
Y_{S, \alpha}^{(n, I)}=f^{(n, I)}\left(\omega ; \mathbf{U}_{\alpha}^{(n)}\right) .
$$

We introduce the generalized dynamic stiffness matrices related to the structure and the internal acoustic fuid:

$$
\begin{aligned}
& {\left[\mathbb{A}_{S}^{(n)}(\omega)\right]=-\omega^{2}\left[M_{S}^{(n)}\right]+i \omega\left[C_{S}^{(n)}\right]+\left[K_{S}^{(n)}\right],} \\
& {\left[\mathbb{A}_{F}^{(n)}(\omega)\right]=-\omega^{2}\left[M_{F}^{(n)}\right]+i \omega\left[C_{F}^{(n)}\right]+\left[K_{F}^{(n)}\right] .}
\end{aligned}
$$

\section{Normalized power spectral density function of the internal noise observation}

In this section, we calculate the normalized power spectral density function of the internal noise observation corresponding to the time-stationary random response of the structural-acoustic system excited by a time-stationary random wall pressure f eld such as a turbulent boundary layer induced by an external fow. This normalized power spectral density function is related to the spatial average of the quadratic mean of the random internal fl id pressure over observation surface $\Sigma$.

\subsection{Random wall pressure f eld excitation}

Let $E$ be the mathematical expectation. Random wall pressure fi ld $p^{+}$applied to external structural surface $\Sigma^{+}$is a second-order real-valued stochastic feld $(\mathbf{x}, t) \mapsto p^{+}(\mathbf{x}, t)$ indexed by $\Sigma^{+} \times \mathbb{R}$ which is centered and mean-square stationary with respect to $t$. In addition, it is assumed that stochastic fiel $p^{+}$is statistically axisymmetric with respect to surface $\Sigma^{+}$and we reuse the model introduced in [17]. Let $\widetilde{R}_{p^{+}}\left(\mathbf{x}, \mathbf{x}^{\prime}, \tau\right)=$ $E\left\{p^{+}(\mathbf{x}, t+\tau) p^{+}\left(\mathbf{x}^{\prime}, t\right)\right\}$ be its real-valued cross-correlation function which is written as $\widetilde{R}_{p^{+}}\left(\mathbf{x}, \mathbf{x}^{\prime}, \tau\right)=$ $\int_{\mathbb{R}} \mathrm{e}^{i \omega \tau} \widetilde{S}_{p^{+}}\left(\mathbf{x}, \mathbf{x}^{\prime}, \omega\right) d \omega$ where $\widetilde{S}_{p^{+}}\left(\mathbf{x}, \mathbf{x}^{\prime}, \omega\right)$ is the 
complex-valued cross-spectral density function [8]. For all $\mathbf{x}$ and $\mathbf{x}^{\prime}$ in $\Sigma^{+}$, we introduce the notation $S_{p^{+}}(\theta-$ $\left.\theta^{\prime}, z, z^{\prime}, \omega\right)=\widetilde{S}_{p^{+}}\left(\mathbf{x}, \mathbf{x}^{\prime}, \omega\right)$ and the power spectral density function of the mean-square stationary stochastic process $\left\{p^{+}(\mathbf{x}, t), t \in \mathbb{R}\right\}$ is a positive-valued function def ned by $\widetilde{\Phi}(\mathbf{x}, \omega)=\widetilde{S}_{p^{+}}(\mathbf{x}, \mathbf{x}, \omega)$ which is independent of $\theta$. Consequently, for all $\mathbf{x}$ fixe in $\Sigma^{+}$, we have $\widetilde{\Phi}(\mathbf{x}, \omega)=\Phi(z, \omega)=S_{p^{+}}(0, z, z, \omega)$. The cross-spectral density function of $p^{+}$is written as [17]

$$
\begin{aligned}
& S_{p^{+}}\left(\theta-\theta^{\prime}, z, z^{\prime}, \omega\right) \\
& \quad=\sqrt{\Phi(z, \omega) \Phi\left(z^{\prime}, \omega\right)} G\left(\xi\left(z, z^{\prime}\right), \eta\left(\theta-\theta^{\prime}, z, z^{\prime}\right), \omega\right),
\end{aligned}
$$

in which $\xi\left(z, z^{\prime}\right)=s^{+}(z)-s^{+}\left(z^{\prime}\right)$ with $s^{+}(z)$ the curvilinear abscissa of generatrix $\Gamma^{+}$introduced in section 2.1, $\eta\left(\gamma, z, z^{\prime}\right)=0.5\left(R^{+}(z)+R^{+}\left(z^{\prime}\right)\right) g(\gamma)$ with $g(\gamma)=\gamma$ if $-\pi \leqslant \gamma \leqslant \pi, g(\gamma)=\gamma-2 \pi$ if $\pi<\gamma \leqslant 2 \pi$ and $g(\gamma)=\gamma+2 \pi$ if $-2 \pi \leqslant \gamma<-\pi$. The complexvalued coherence function $G(\xi, \eta, \omega)$ is given by the Corcos model [2] which is written as

$$
G(\xi, \eta, \omega)=\exp \left\{i \frac{\xi \omega}{U_{c}}-\frac{|\xi|}{L_{1}(\omega)}-\frac{|\eta|}{L_{2}(\omega)}\right\}
$$

in which $U_{c}=0.65 U_{E}$ is the average convection velocity with $U_{E}$ the average external fow velocity. The longitudinal and lateral correlation scales $L_{1}(\omega)$ and $L_{2}(\omega)$ are written as $L_{1}(\omega)=U_{c} /(0.115|\omega|)$ and $L_{2}(\omega)=$ $U_{c} /(0.7|\omega|)$. For the application presented in section 7 , the model used for the power spectral density function $\Phi(z, \omega)$ is written as

$$
\begin{aligned}
\Phi(z, \omega)= & \frac{1}{4000} \rho_{0}^{2} U_{E}^{4} \delta(z)^{3} \\
& \times \omega^{2}\left(U_{E}^{2}+25 \omega^{2} \delta(z)^{2}\right)^{-3 / 2},
\end{aligned}
$$

in which $\delta(z)$ is the thickness displacement of the boundary layer.

\subsection{Reference power spectral density function}

Let $s_{p_{\text {ref }}^{+}}(\omega)$ be the reference power spectral density function related to surface $\Sigma^{+}$. Let $\Pi_{\Sigma^{+}}$be the spatial average over surface $\Sigma^{+}$of the mean power of stochastic process $\left\{p^{+}(\mathbf{x}, t), t \in \mathbb{R}\right\}$ for $\mathbf{x}$ in $\Sigma^{+}$. We then have $\Pi_{\Sigma^{+}}=\int_{\mathbb{R}} s_{p_{\text {ref }}^{+}}(\omega) d \omega$ in which $s_{p_{\text {ref }}^{+}}(\omega)$ is the reference power spectral density function def ned by

$$
s_{p_{\text {ref }}^{+}}(\omega)=\frac{1}{\left|\Sigma^{+}\right|} \int_{\Sigma^{+}} \widetilde{\Phi}(\mathbf{x}, \omega) d \Sigma^{+}(\mathbf{x}) .
$$

\subsection{Normalized power spectral density function calculation}

From equations (37) and (50), we deduce that

$$
\begin{aligned}
Y_{S, \alpha}^{(n, I)}(t)= & -\int_{\Gamma^{+}} \int_{0}^{2 \pi} p^{+}(\theta, z, t) \\
& \times F^{(n, I)}(\theta) d \theta\left\langle\mathbf{U}_{\alpha}^{(n)}, \mathbf{n}\right\rangle(z) d \Gamma^{+}(z),
\end{aligned}
$$

in the time domain. Therefore, $\mathbf{Y}_{S}^{(n, I)}(t)=\left(Y_{S, 1}^{(n, I)}(t)\right.$, $\left.\ldots, Y_{S, N_{S}^{(n)}}^{(n, I)}(t)\right)$ is a second-order, centered, mean-square stationary and mean-square continuous stochastic process indexed by $\mathbb{R}$ with values in $\mathbb{R}^{N_{S}^{(n)}}$. The cross-correlation function of stochastic processes $Y_{S, \alpha}^{(n, I)}(t)$ and $Y_{S, \alpha^{\prime}}^{\left(n^{\prime}, I^{\prime}\right)}(t)$ is def ned by

$$
R_{Y_{S, \alpha} Y_{S, \alpha^{\prime}}}^{\left(n, n^{\prime}, I, I^{\prime}\right)}(\tau)=E\left\{Y_{S, \alpha}^{(n, I)}(t+\tau) Y_{S, \alpha^{\prime}}^{\left(n^{\prime}, I^{\prime}\right)}(t)\right\},
$$

and can be written as

$$
R_{Y_{S, \alpha} Y_{S, \alpha^{\prime}}}^{\left(n, n^{\prime}, I, I^{\prime}\right)}(\tau)=\int_{\mathbb{R}} \mathrm{e}^{i \omega \tau} S_{Y_{S, \alpha} Y_{S, \alpha^{\prime}}}^{\left(n, n^{\prime}, I, I^{\prime}\right)}(\omega) d \omega,
$$

where $S_{Y_{S, \alpha} Y_{S, \alpha^{\prime}}}^{\left(n, n^{\prime} I, I^{\prime}\right)}(\omega)$ is the cross-spectral density function which can be written as

$$
\begin{aligned}
& S_{Y_{S, \alpha} Y_{S, \alpha^{\prime}}}^{\left(n, n^{\prime}, I, I^{\prime}\right)}(\omega) \\
& \quad=2 \pi \delta_{n n^{\prime}} \delta_{I I^{\prime}}\left(1+\delta_{0 n}\right) \\
& \quad \times \int_{\Gamma^{+}} \int_{\Gamma^{+}} \int_{0}^{\pi} S_{p^{+}}\left(\gamma, z, z^{\prime}, \omega\right) \cos n \gamma d \gamma \\
& \quad \times\left\langle\mathbf{U}_{\alpha}^{(n)}, \mathbf{n}\right\rangle(z)\left\langle\mathbf{U}_{\alpha^{\prime}}^{\left(n^{\prime}\right)}, \mathbf{n}\right\rangle\left(z^{\prime}\right) d \Gamma^{+}(z) d \Gamma^{+}\left(z^{\prime}\right) .
\end{aligned}
$$

The matrix-valued spectral density function $\left[S_{\mathbf{Y}_{S}}^{(n)}(\omega)\right]$ of vector-valued process $\mathbf{Y}_{S}^{(n, I)}(t)$ is then defi ed by $\left[S_{\mathbf{Y}_{S}}^{(n)}(\omega)\right]_{\alpha \alpha^{\prime}}=S_{Y_{S, \alpha} Y_{S, \alpha^{\prime}}}^{(n, n, I, I)}(\omega)$.

Let $\Pi_{\Sigma}$ be the spatial average over surface $\Sigma$ of the mean power of stochastic process $\{p(\mathbf{x}, t), t \in \mathbb{R}\}$ for $\mathbf{x}$ in $\Sigma$. From this def nition, we deduce that $\Pi_{\Sigma}=$ $E\left\{\frac{1}{|\Sigma|} \int_{\Sigma} p(\mathbf{x}, t)^{2} d \Sigma\right\}$ which can be rewritten as

$$
\Pi_{\Sigma}=\int_{\mathbb{R}} s_{p_{\Sigma}}(\omega) d \omega,
$$

where $s_{p_{\Sigma}}(\omega)$ is the power spectral density function of the internal noise observation. It is proved $[10,17,18]$ that

$$
s_{p_{\Sigma}}(\omega)=s_{0}(\omega)+\sum_{n=0}^{+\infty} s_{p_{\Sigma}}^{(n)}(\omega) .
$$


The term $s_{0}(\omega)$ is an additional function induced by the presence of $-\kappa \pi_{2}$ in equation (5), whose contribution exists only for $n=0$ and which is written as

$$
\begin{aligned}
s_{0}(\omega)= & \frac{8 \pi^{2} \kappa}{|\Sigma|} \operatorname{tr}\left\{\pi \kappa\left[S_{\mathbf{X}_{S}}^{(n)}(\omega)\right]\left[U_{n}^{(n)}\right]\right. \\
& \left.-\omega \rho_{0} \Im m\left(\left[S_{\mathbf{X}_{S F}^{(n)}}(\omega)\right]\left[\Upsilon_{n}^{(n)}\right]\right)\right\},
\end{aligned}
$$

in which $\Im m$ is the imaginary part, $\operatorname{tr}$ is the trace operator and $\left[\Upsilon_{n}^{(n)}\right]$ is the matrix defi ed by

$$
\left[\Upsilon_{n}^{(n)}\right]_{\beta \alpha}=\int_{\Gamma} \Psi_{\beta}^{(n)} \int_{\Gamma^{-}}\left\langle\mathbf{U}_{\alpha}^{(n)}, \mathbf{n}\right\rangle d \Gamma^{-} d \Gamma .
$$

The main contributions are the terms $s_{p_{\Sigma}}^{(n)}(\omega)$ which are written as

$$
s_{p_{\Sigma}}^{(n)}(\omega)=2 \pi \frac{\omega^{2} \rho_{0}^{2}}{|\Sigma|} \operatorname{tr}\left\{\left[S_{\mathbf{X}_{F}}^{(n)}(\omega)\right]\left[\Psi^{(n)}\right]\right\} .
$$

In equations (63) and (65), we have

$$
\begin{aligned}
& {\left[S_{\mathbf{X}_{F}}^{(n)}(\omega)\right]=} {\left[H_{F S}^{(n)}(\omega)\right]\left[S_{\mathbf{Y}_{S}}^{(n)}(\omega)\right]\left[H_{F S}^{(n)}(\omega)\right]^{*}, } \\
& {\left[S_{\mathbf{X}_{S}}^{(n)}(\omega)\right]=} {\left[H_{S}^{(n)}(\omega)\right]\left[S_{\mathbf{Y}_{S}}^{(n)}(\omega)\right]\left[H_{S}^{(n)}(\omega)\right]^{*}, } \\
& {\left[S_{\left.\mathbf{X}_{S F}^{(n)}(\omega)\right]=}\left[H_{S}^{(n)}(\omega)\right]\left[S_{\mathbf{Y}_{S}}^{(n)}(\omega)\right]\left[H_{F S}^{(n)}(\omega)\right]^{*},\right.} \\
& {\left[H_{S}^{(n)}(\omega)\right]=}\left(\left[\mathbb{A}_{S}^{(n)}(\omega)\right]+\kappa\left[J^{(n)}\right]-\omega^{2}\left[A_{F}^{(n)}\right]\right. \\
&\left.\times\left[\mathbb{A}_{F}^{(n)}(\omega)\right]^{-1}\left[A_{F}^{(n)}\right]^{T}\right)^{-1}, \\
& {\left[H_{F S}^{(n)}(\omega)\right]=} i \omega\left[\mathbb{A}_{F}^{(n)}(\omega)\right]^{-1}\left[A_{F}^{(n)}\right]^{T}\left[H_{S}^{(n)}(\omega)\right], \\
& {\left[H_{S F}^{(n)}(\omega)\right]^{*}=}-\left[H_{F S}^{(n)}(\omega)\right], \\
& {\left[\Psi^{(n)}\right]_{\beta \beta^{\prime}}=} \int_{\Gamma} \Psi_{\beta}^{(n)} \Psi_{\beta^{\prime}}^{(n)} d \Gamma \\
& {\left[U_{n}^{(n)}\right]_{\alpha \alpha^{\prime}}=} \int_{\Gamma} \int_{\Gamma^{-}}\left\langle\mathbf{U}_{\alpha}^{(n)}, \mathbf{n}\right\rangle d \Gamma^{-} \\
& \times \int_{\Gamma^{-}}\left\langle\mathbf{U}_{\alpha^{\prime}}^{(n)}, \mathbf{n}\right\rangle d \Gamma^{-} d \Gamma .
\end{aligned}
$$

Finally, the normalized power spectral density function of the internal noise observation is def ned by

$$
s_{p_{\Sigma}, \mathrm{norm}}(\omega)=\frac{s_{p_{\Sigma}}(\omega)}{s_{p_{\text {ref }}^{+}}(\omega)} .
$$

It should be noted that $\left[\mathbb{A}_{F}^{(n)}(\omega)\right]$ is a diagonal matrix and consequently, the inverse matrix which appears in equations (69) and (70) is explicitly known.

\section{Shape optimization with respect to the aspect ratio of the dome}

\subsection{Class of geometry}

The objective is to optimize the dome shape in order to minimize the noise related to observation surface $\Sigma$ located inside the internal acoustic cavity. Since we are looking for the inf uence of the dome curvature, all the other main parameters of the structural acoustic system (volume of the internal acoustic cavity, external structural surface area, dome thickness and constitutive material) have to remain constant when the shape of the dome is modif ed in the dome-shape optimization process. The external power injected in the structural-acoustic system is proportional to the external structural surface area on which the external random wall pressure feld excitation is applied. Since we are only interested in analyzing the inf uence of the dome curvature on the internal noise, this surface area has to remain a constant in order to not introduce a strong variation of the external power input when the dome shape is modif ed. The volume of the internal acoustic cavity is a constant for that the modal density of the internal acoustic cavity be nearly a constant when the dome shape is modif ed. In addition, since the dome thickness is very small compared to the other structural-acoustic system dimensions, the area of internal structural surface $\Sigma^{-}$is almost equal to the area of external structural surface $\Sigma^{+}$. The internal generatrix $\Gamma^{-} \cup \Gamma_{F}^{-}$is chosen as an arc of an ellipse, possibly extended by a line segment of a line parallel to axis $(0 z)$ and belonging to the generative plane. This ellipse is centered in the reference system origin and characterized by its semiminor axis which has a fxed value $a_{\text {ref }}$ and its semimajor axis $b$ (see figu es 3 and 4 ). The characteristic ratio of the ellipse is defi ed by $q=b / a_{\text {ref. }}$. Each value of characteristic ratio $q$ define a geometric conf guration of the structural-acoustic system and $q$ is called the structural aspect ratio. Figures 3 and 4 def ne the $z$-axes, denoted as $z_{a}, z_{b}, z_{c}, z_{d}=b$ and $z_{e}$, of all

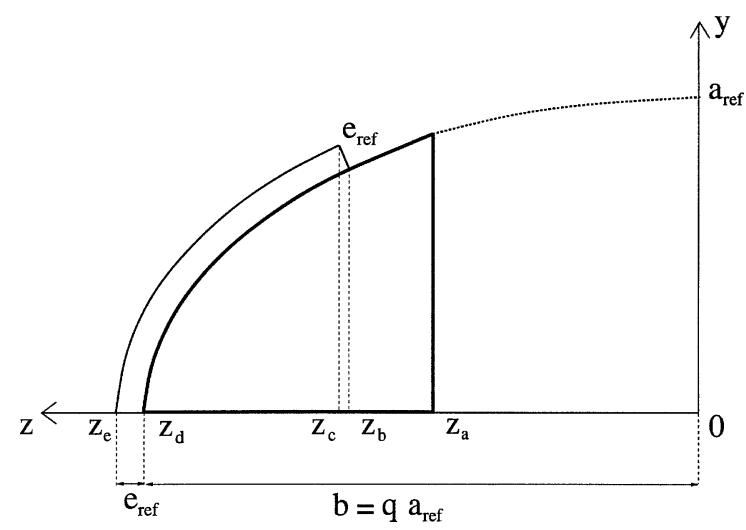

Figure 3. Geometry of the generative plane: case of an arc of the ellipse. 


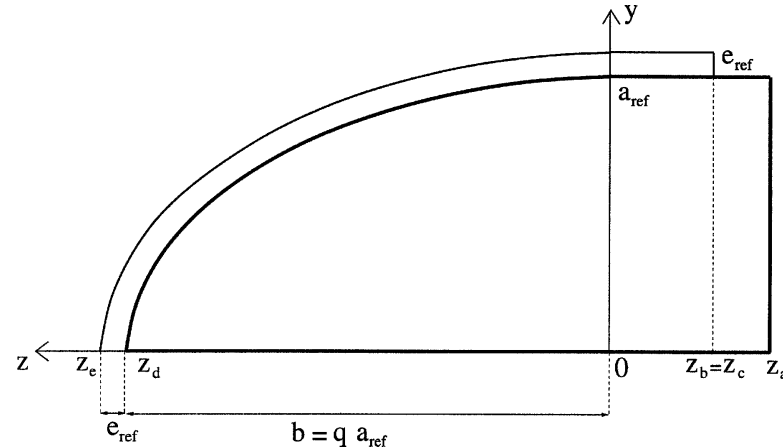

Figure 4. Geometry of the generative plane: case of a quarter of the ellipse extended by a line segment.

the particular points which allow the geometry of the structure and the internal acoustic cavity to be def ned. Figures 3 and 4 correspond to $z_{a}>0$ (an arc of the ellipse) and $z_{a}<0$ (a quarter of the ellipse extended by a line segment) respectively. The internal acoustic cavity is located between $z_{a}$ and $z_{d}=b$. The length of the internal acoustic cavity is denoted as $\ell$ and is such that $\ell=z_{d}-z_{a}=b-z_{a}$. The constraint $\left|\Sigma^{-}\right|$ remains a constant def ned by the value of $z_{b}$. A reference conf guration is def ned by a given value $q_{\mathrm{ref}}>1$ of structural aspect ratio $q$. For all values of $q$, the structure thickness is a constant $e_{\text {ref }}$ corresponding to the reference conf guration. We have $z_{e}=z_{d}+e_{\text {ref }}$.

\subsection{Optimization problem with respect to the aspect ratio of the dome}

\subsubsection{Optimization parameter}

Structural aspect ratio $q>0$ takes its values in an interval denoted as $\mathcal{Q}$ and define by

$$
\mathcal{Q}=] 0,2 q_{\text {ref }}[.
$$

The values of $q$ such that $0<q<1, q=1$ and $1<$ $q<2 q_{\text {ref }}$ correspond to a dome which is not slender, one which is part of a sphere, and one which is a slender structure respectively.

\subsubsection{Cost function}

The analysis is made over the frequency bands $B_{1}=$ $[1,1500] \mathrm{Hz}$ and $B_{2}=[1,500] \mathrm{Hz}$. Given index $i=1,2$, the cost function $q \mapsto J_{\Sigma}^{(i)}(q)$ from $\mathcal{Q}$ into $] 0,+\infty[$ is define for each frequency band $B_{i}$ by

$$
J_{\Sigma}^{(i)}(q)=\frac{1}{\left|B_{i}\right|} \int_{B_{i}} s_{p_{\Sigma}, \operatorname{norm}}(\omega) d \omega,
$$

in which $s_{p_{\Sigma} \text {,norm }}(\omega)$ is the normalized power spectral density function related to the observation surface $\Sigma$ and which is def ned by equation (73) as a function of structural aspect ratio $q$.

\subsubsection{Optimization problem}

Given the frequency band $B_{i}$, the optimization problem associated with cost function $q \mapsto J_{\Sigma}^{(i)}(q)$ consists in f nding $q^{(i)}$ in $\mathcal{Q}$ such that

$$
J_{\Sigma}^{(i)}\left(q^{(i)}\right)=\min _{q \in \mathcal{Q}} J_{\Sigma}^{(i)}(q) .
$$

\subsubsection{Solution method and structural shape parametric optimization}

Each value of $q$ in $\mathcal{Q}$ def nes a geometric conf guration which has to be modeled by the f nite element method. Consequently, for each given structural aspect ratio $q$, a finit element mesh of the dome and a f nite element mesh of the internal acoustic cavity have to be constructed, then the structural modes and the acoustic modes have to be calculated before starting to compute the cost function for the value of $q$ considered. It should be noted that the cost function is strongly nonlinear with respect to parameter $q$ and a sensitivity analysis [6] is not adapted to the problem under consideration. For a given value of $q$, an examination of the numerical cost induced by computation of the cost function (and possibly of its derivative with respect to $q$ ) shows that the use of an automatic optimization algorithm [4,14-16,19] would lead to a very high numerical cost, probably with some serious numerical diff culties. In addition, we are interested not only in the optimum value of the structural aspect ratio but also in performing the structural shape parametric optimization; that is to say, in knowing the variation of the cost function as a function of $q$ in order to identify the domain of $q$ for which the internal noise could be high. Consequently, we have chosen a very simple method (which is perfectly adapted to the problem and very eff cient in this case), consisting in constructing the graph of the cost function $q$ by $q$ and then identifying the minimun value of the cost function by looking at its graph. Taking into account the high numerical cost for computing one value of the cost function, interval $\mathcal{Q}$ is discretized by a 'reasonable' number of discrete values of $q$.

\section{Numerical results}

\subsection{Numerical data}

\subsubsection{Structure}

The structure is made of a composite material modeled as a linear isotropic homogeneous material with density $\rho_{S}=1750 \mathrm{~kg} / \mathrm{m}^{3}$, Young's modulus $E_{S}=1.615$. $10^{10} \mathrm{~N} / \mathrm{m}^{2}$, Poisson's ratio $\nu_{S}=0.137$ and dissipation coefficien $\xi_{S}=0.02$. 
The reference geometric configuratio is define by $a_{\mathrm{ref}}=3.13 \mathrm{~m}, q_{\mathrm{ref}}=3.6$ (therefore, $b_{\text {ref }}=11.26 \mathrm{~m}$ ), $l_{\text {ref }}=4.29 \mathrm{~m}$, structure thickness $e_{\text {ref }}=0.027 \mathrm{~m}$ and $\left|\Sigma^{+}\right|_{\text {ref }}=50.6 \mathrm{~m}^{2}$. For each value of $q$, a finit element mesh of the structure (generative plane) is constructed using parabolic $\mathrm{f}$ nite elements with 8 nodes. The thickness (smallest dome dimension) is discretized into two f niteelement layers. The $f$ nite-element type and the number of layers were determined from studing the result of convergence of all the eigenfunctions and eigenvalues corresponding to the extreme conf guration $q=7.0$ which leads to the greatest number of modes within frequency band $B_{0}=[1,2000] \mathrm{Hz}$. For the extreme geometric conf gurations corresponding to $q=0.3$ and $q=7.0$, the number of $f$ nite elements and the number of nodes are $616-2469$ and $892-3573$ respectively.

\subsubsection{Internal acoustic flud}

The internal acoustic $\mathrm{fl}$ id is water with density $\rho_{0}=$ $1000 \mathrm{~kg} / \mathrm{m}^{3}$, speed of sound $c_{0}=1500 \mathrm{~m} / \mathrm{s}$ and dissipation coeff cient $\xi_{F, \beta}^{(n)}=0.01$ for every acoustic mode. The acoustic cavity volume $\left|D_{F}\right|_{\text {ref }}$ of the reference geometric conf guration is assigned the value $64 \mathrm{~m}^{3}$. For each value of $q$, a finit element mesh (compatible with the structure) of the internal acoustic cavity (generative plane) is constructed using linear triangular $f$ nite elements. For the extreme geometric conf gurations corresponding to $q=0.3$ and $q=7.0$, the number of $\mathrm{f}$ nite elements and the number of nodes are 8694-4499 and 13663-7073 respectively. The uniform distance between boundary $\Sigma^{-} \cup \Sigma_{F}^{-}$and observation surface $\Sigma$ is equal to $0.6 a_{\text {ref }}$.

\subsubsection{Data for the excitation corresponding to a random wall pressure fie d}

The parameter values of the model define by equations (53)-(55) are $U_{E}=7.5 \mathrm{~m} / \mathrm{s}$ and $\delta(z)=\delta_{\max }\left(z_{e}-\right.$ $z) /\left(z_{e}-z_{c}\right)$ with $\delta_{\max }=5.1 \cdot 10^{-3} \mathrm{~m}$.

\subsection{Structural shape parametric optimization results}

In order to decrease the numerical cost due to the optimization procedure, the non usual additional terms induced by the presence of $-\kappa \pi_{2}$ in equation (5), whose contribution exists only for $n=0$, are not taken into account in the numerical calculation. It should be noted that the coupling terms (see equations (26) and (27)) between the structure and the internal acoustic cavity, represented by bilinear form $a_{F}^{(n)}(\Psi, \mathbf{U})$ def ned by equation (34), are always kept in the numerical calculation.

\subsubsection{Eigenmode extraction and convergence computation}

The f nite element meshes used for the structure and the internal acoustic cavity allow us to compute the eigen- modes whose associated eigenfrequencies are in the frequency band [1,2000] Hz. The structural modes and acoustic modes are computed by the subspace iteration method [1] checking Sturm's sequence, using an accelerated scheme for convergence and a Lanczos method for initialization. For each circumferential wave number $n$, the reduced matrix model uses the structural modes and acoustic modes whose eigenfrequencies are in frequency band $B_{0}=[1,2000] \mathrm{Hz}$; this ensures that power spectral density functions $\omega \mapsto s_{p_{\Sigma}}^{(n)}(\omega)$ def ned by equation (65) are uniformly converged over frequency band $B_{1}=[1,1500] \mathrm{Hz}$. An analysis was carried out [10] to study the convergence of normalized power spectral density function $s_{p_{\Sigma} \text {,norm }}$ def ned by equation (73) with respect to circumferential wave number $n$. This analysis shows that uniform convergence over frequency band $B_{1}=[1,1500] \mathrm{Hz}$ is achieved when contributions $n=$ $0,1,2,3,4,5$ are taken into account. For instance, fig ure 5 shows the variation of $\mapsto J_{\Sigma}^{(1)}(1)$ as a function of $n$.

\subsubsection{Optimization results}

Let us introduce the frequency moving average of normalized power spectral density function $s_{p_{\Sigma} \text {,norm, }}$ def ned by

$$
\left\langle s_{p_{\Sigma}, \text { norm }}\right\rangle_{\Delta f}(\omega)=\frac{1}{\Delta \omega} \int_{\omega-\Delta \omega / 2}^{\omega+\Delta \omega / 2} s_{p_{\Sigma}, \text { norm }}\left(\omega^{\prime}\right) d \omega^{\prime},
$$

in which $\Delta \omega=2 \pi \Delta f$. The following numerical results were obtained [10] for $\Delta f=100 \mathrm{~Hz}$ : figu es 6 to 9 show the graphs of functions $\omega \mapsto\left\langle s_{p_{\Sigma}} \text {, norm }\right\rangle_{\Delta f}(\omega)$ expressed in $\mathrm{Hz}$ for several values of structural aspect ratio $q$; figu es 10 and 11 show the graphs of cost functions $q \mapsto$ $J_{\Sigma}^{(1)}(q)$ and $q \mapsto J_{\Sigma}^{(2)}(q)$ for frequency bands $B_{1}$ and $B_{2}$ respectively. It can be seen that for the two frequency

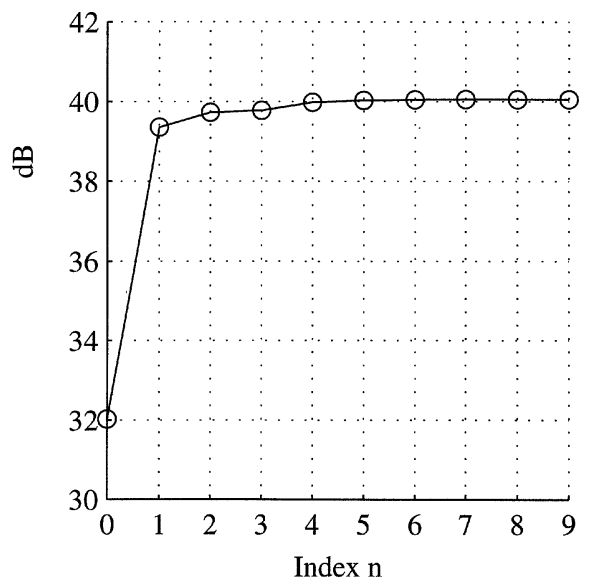

Figure 5. Convergence: variation of $10 \times \log _{10}\left(J_{\Sigma}^{(1)}(1) /\right.$ $10^{-18}$ ) as a function of $n$. 


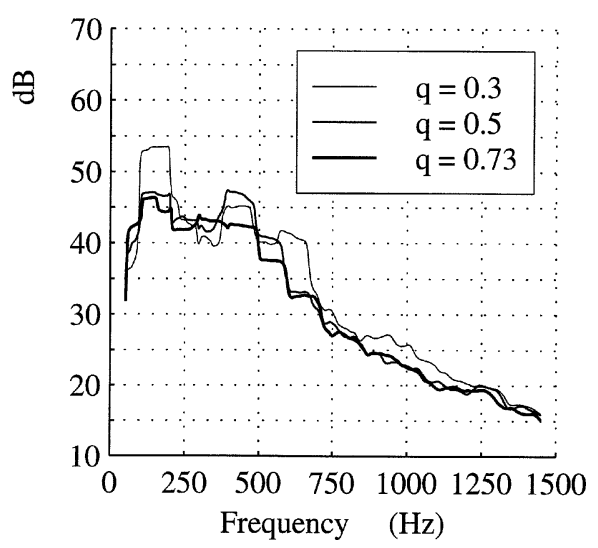

Figure 6. Graphs of functions $\omega \mapsto 10 \times \log _{10}\left(\left\langle s_{p_{\Sigma}, \text { norm }}\right\rangle_{100}(\omega)\right.$ $/ 10^{-18}$ ) for structural aspect ratio $q=0.3$ (thin solid line), 0.5 (medium solid line), 0.73 (thick solid line).

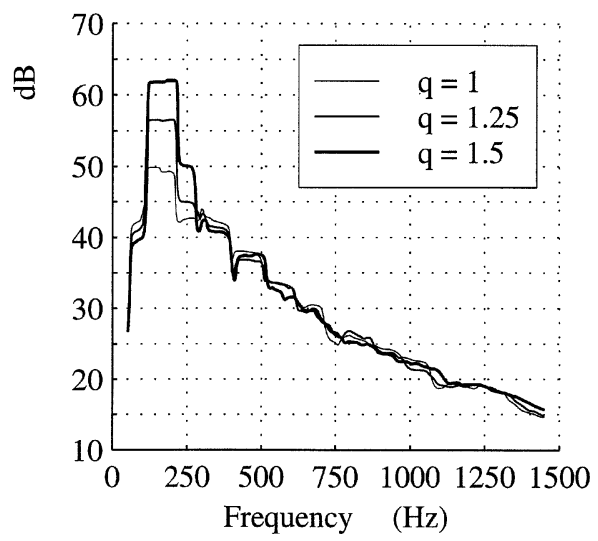

Figure 7. Graphs of functions $\omega \mapsto 10 \times \log _{10}\left(\left\langle s_{p_{\Sigma}} \text {, norm }\right\rangle_{100}(\omega)\right.$ $/ 10^{-18}$ ) for structural aspect ratio $q=1.0$ (thin solid line), 1.25 (medium solid line), 1.5 (thick solid line).

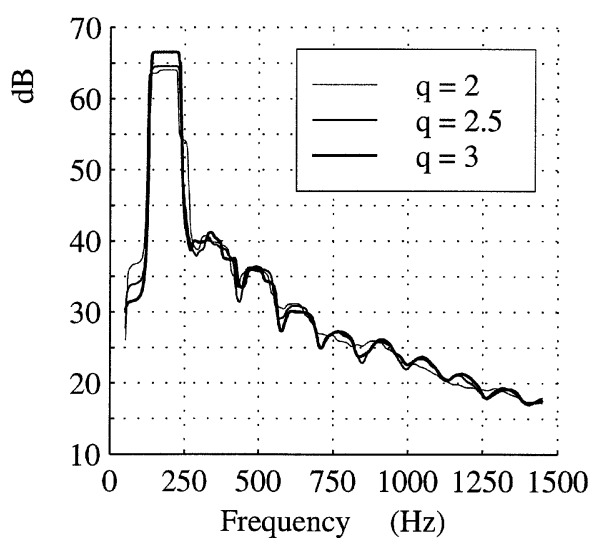

Figure 8. Graphs of functions $\omega \mapsto 10 \times \log _{10}\left(\left\langle s_{p_{\Sigma}, \text { norm }}\right\rangle_{100}(\omega)\right.$ $/ 10^{-18}$ ) for structural aspect ratio $q=2.0$ (thin solid line), 2.5 (medium solid line), 3.0 (thick solid line).

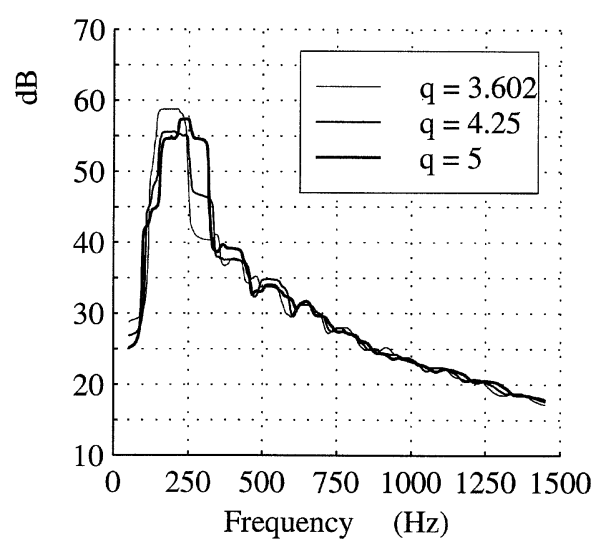

Figure 9. Graphs of functions $\omega \mapsto 10 \times \log _{10}\left(\left\langle s_{p_{\Sigma}}, \text { norm }\right\rangle_{100}(\omega)\right.$ $/ 10^{-18}$ ) for structural aspect ratio $q=3.602$ (thin solid line), 4.25 (medium solid line), 5.0 (thick solid line).

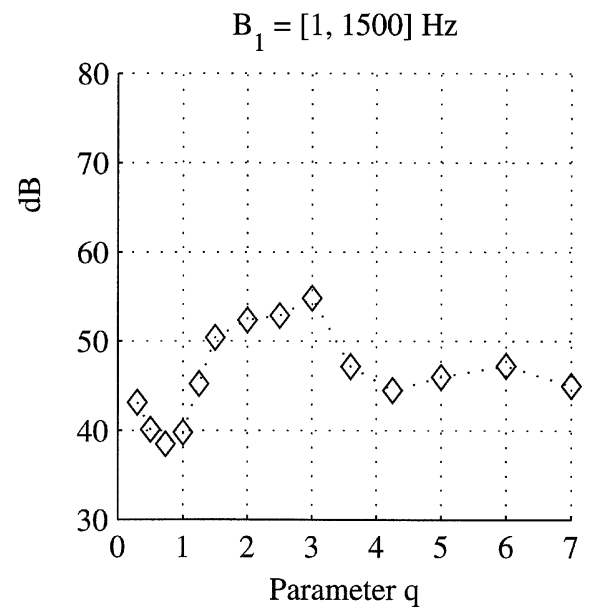

Figure 10. Graph of cost function $q \mapsto 10 \times \log _{10}\left(J_{\Sigma}^{1)}(q) /\right.$ $10^{-18}$ ) for frequency band $B_{1}=[1,1500] \mathrm{Hz}$.

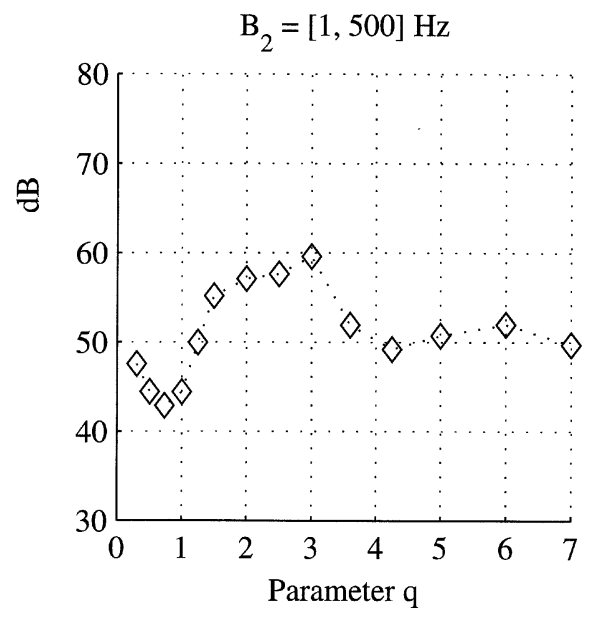

Figure 11. Graph of cost function $q \mapsto 10 \times \log _{10}\left(J_{\Sigma}^{2)}(q) /\right.$ $10^{-18}$ ) for frequency band $B_{2}=[1,500] \mathrm{Hz}$. 
bands, the lowest energy level occurs when $q=0.73$. Nevertheless, it should be noted that the minimum value obtained for $q=0.73$ differs by only about $1 \mathrm{~dB}$ from the energy level obtained for $q=1.0$. The highest energy levels are obtained for $q$ belonging to the range [1.5, 3.5].

\section{Conclusions}

The analysis presented in this paper shows that the structural shape optimization of a dome with respect to its aspect ratio, excited by an external random wall pressure fiel, has a clear solution which minimizes internal noise in the low- and medium-frequency ranges. This solution corresponds to structural aspect ratios belonging to subinterval $[0.7,1.0]$. In addition, this analysis shows that internal noise is high in the low- and mediumfrequency ranges when the structural aspect ratio belongs to subinterval $[1.5,3.5]$ which should be avoided.

\section{References}

[1] Bathe K.J., Wilson E.L., Numerical Methods in Finite Element Analysis, Prentice Hall, New York, 1976.

[2] Corcos G.M., The structure of the turbulent pressure f eld in boundary layer fows, J. Fluid Mech. 18 (1964) 353378.

[3] Fahy F., Sound and Structural Vibration, Academic Press, London, 1987.

[4] Fletcher R., Practical Methods of Optimization - Vol. 2, Constrained Optimization, Wiley, New York, 1980.

[5] Haslinger J., Neittaanmaki P., Finite Element Approximation for Optimal Shape, Material and Topology Design, Second Edition, John Wiley, Chichester, 1996.

[6] Haug E.J., Choi K.K., Komkov V., Design Sensitivity Analysis of Structural Systems, Academic Press, 1986.
[7] Junger M.C., Feit D., Sound, Structures and their Interaction, Acoust. Soc. Am. Publications on Acoustics, Woodbury, 1993 (originally published MIT Press, Cambridge, 1972).

[8] Krée P., Soize C., Mathematics of Random Phenomena, Reidel, Dordrecht, 1983.

[9] Lesueur C., Rayonnement Acoustique des Structures, Eyrolles, Paris, 1988.

[10] Michelucci J.-C., Optimisation de forme structurale axisymétrique en vibroacoustique interne dans les domaines des basses et moyennes fréquences, Doctorat de l'Ecole Centrale Paris, 1998.

[11] Morand H.J.-P., Ohayon R., Fluid Structure Interaction, Wiley, New York, 1995.

[12] Ohayon R., Soize C., Structural Acoustics and Vibration, Academic Press, London, 1998.

[13] Pierce A.D., Acoustics: An Introduction to its Physical Principles and Applications, Acoust. Soc. Am. Publications on Acoustics, Woodbury, 1989 (originally published McGraw-Hill, New York, 1981).

[14] Polak E., Computational Methods in Optimization, Academic Press, New York, 1971.

[15] Powell M.J.D., Nonlinear Optimization, Academic Press, New York, 1981.

[16] Sewell M.J., Maximum and Minimum Principles, Cambridge University Press, Cambridge, 1987.

[17] Soize C., Desanti A., David J.-M., Dynamic and acoustic response of coupled structure-dense f uid axisymmetric systems excited by a random wall pressure feld, Rech. Aerospatiale 5 (1989) 1-13.

[18] Soize C., The Fokker-Planck Equation for Stochastic Dynamical Systems and its Explicit Steady State Solutions, World Scientif c, Singapore, 1994.

[19] Walter E., Pronzato L., Identif cation of Parametric Models from Experimental Data, Springer-Verlag, Berlin, 1997. 